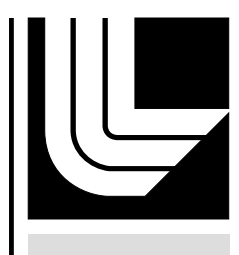

LAW RENCE LIVERMORE N A T IO N A L LABORATORY

\title{
UCRL-TR-224230
}

\section{Combining Equations of State in Kull}

M. Ulitsky, G. Zimmerman, P. Renard

September 7, 2006 


\section{Legal Notices}

Copyright 2003 Regents of the University of California

This work was produced at the University of California, Lawrence Livermore National Laboratory (UC LLNL) under contract no. W-7405-ENG-48 (Contract 48) between the U.S. Department of Energy (DOE) and The Regents of the University of California (University) for the operation of UC LLNL. The rights of the Federal Government are reserved under Contract 48 subject to the restrictions agreed upon by the DOE and University as allowed under DOE Acquisition Letter 97-1.

\section{DISCLAIMER}

This work was prepared as an account of work sponsored by an agency of the United States Government. Neither the United States Government nor the University of California nor any of their employees, makes any warranty, express or implied, or assumes any liability or responsibility for the accuracy, completeness, or usefulness of any information, apparatus, product, or process disclosed, or represents that its use would not infringe privately-owned rights. Reference herein to any specific commercial products, process, or service by trade name, trademark, manufacturer or otherwise does not necessarily constitute or imply its endorsement, recommendation, or favoring by the United States Government or the University of California. The views and opinions of authors expressed herein do not necessarily state or reflect those of the United States Government or the University of California, and shall not be used for advertising or product endorsement purposes. 
UCRL-TR-?

\section{Combining Equations of State in Kull}

Mark Ulitsky, George Zimmerman, Paul Renard

Lawrence Livermore National Laboratory 



\section{Contents}

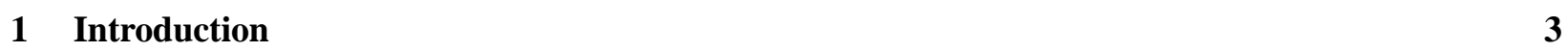

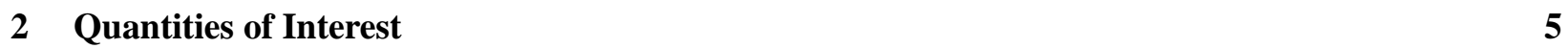

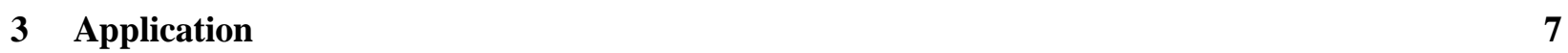

\begin{tabular}{|lr|}
\hline Analvtic $Z_{\rho f f}$ & 8
\end{tabular}

5 Thermodvnamic Consistency 10

6 Test Case $\quad 12$ 


\section{Introduction}

For ICF applications, the temperatures are hot enough that materials can transition to an atomic state or plasma. If we are simulating an ICF capsule, then either thru ALE, physical diffusion, transmutation by nuclear reactions, a mix model, or numerical diffusion (if we are running an Eulerian code), we will generate zones that contain multiple materials. It may be desired to treat certain mixtures of materials or mixed zones as atomic mixtures rather than as chunk mixtures. For example, suppose we have a deuterated material that is initially separated from a tritiated material. As these quantities come into contact at the atomic level, high energy neutrons will be generated from the $D+T$ reaction. However, if we had a chunk of deuterium and a chunk of tritium in the same computational zone, then the $D+T$ reaction would not take place.

In dealing with atomic mixtures, two topics that immediately come to mind are mixed equations of state and mixed opacities. This report will only focus on the equation of state (EOS) aspect and its implementation in the Kull code. Imagine we have a zone that contains an atomic mixture of plastic and steel. If we know the density, temperature, and isotopics of this mixture, then a natural question is how will we compute the pressure and specific internal energy of the mixture as well as the derivatives of these quantities with respect to density and temperature. Let's consider the case where we have tabular thermodynamic data for plastic and steel (as a function of density and temperature), and our goal is to determine how to use these tables to compute the thermodynamic quantities of interest.

The first step is to decide a priori which isotopes should be associated with which table (we will let table 1 refer to the plastic table and table 2 refer to the steel table). This step is necessary so that we can compute mass fractions for each table. Let's assume that the plastic consists of isotopes of hydrogen and carbon, while the steel consists of iron, manganese, and zinc. We can then use a simple Z-range (here, Z refers to the atomic number of the element in question) to decide which isotopes go where. For example, table 1 could have a Z-range of $(0,10.5)$ so that it captures all of the hydrogen and carbon isotopes and table 2 could have a range of $(10.5,50.5)$ to capture all of its higher $Z$ elements. Since we have access to the mixture density, number fractions and number density, we can readily convert the number fractions to mass fractions $\left(y_{i}\right)$, where they are defined as $y_{1} \equiv M_{\text {plastic }} /\left(M_{\text {plastic }}+M_{\text {steel }}\right)$ and $y_{2} \equiv M_{\text {steel }} /\left(M_{\text {plastic }}+M_{\text {steel }}\right)$.

The second step is to bring the atomic mixture into equilibrium through a Newton-Raphson iteration scheme and solve for the sub-densities, $\rho_{i}$, defined as $\rho_{1} \equiv M_{\text {plastic }} / V_{\text {plastic }}$ and $\rho_{2} \equiv M_{\text {steel }} / V_{\text {steel }}$. In many instances it will be more useful to work with specific volume, $v_{i}$, which is simply the reciprocal of the density, and we will use whichever qunantity will make a particular derivation more transparent. An important constraint in the Newton-Raphson procedure is that the mixture density remains unchanged by the iteration procedure. Therefore, for an $\mathrm{N}$ material example, we would have the specific volumes satisfying:

$$
v=\frac{1}{\rho}=\sum_{i=1}^{N} y_{i} v_{i} .
$$

By equilibration, we mean that we iterate on the sub-densities until

$$
f=f_{1}\left(\rho_{1}, T\right)=f_{2}\left(\rho_{2}, T\right)=\ldots=f_{N}\left(\rho_{N}, T\right),
$$

where $f$ is the quantity we want to equilibrate. Here the subscript under the $f$ refers to the particular table that is used to perform the thermodynamic lookup (for notational simplicity, we will usually omit this subscript in future equations). Also, for mathematical well-posedness, $f\left(\rho_{i}, T\right)$ should be monotonic in $\rho_{i}$ to obtain unique solutions.

In Kull, there are four different equilibrate options. We can let $f$ be the total pressure, chemical potential, analytic electron density, or tabular electron density. The electron density, $n_{e}$, is directly related to a quantity known as the effective charge or $Z_{\text {eff }}$ by the simple relation $n_{e}=Z_{e f f} * \rho / A$, where $A$ is the average atomic weight. We can compute $Z_{\text {eff }}$ using an analytic formula based on Thomas-Fermi theory or we can query 
the EOS table to return a value for $Z_{e f f}$. Hence, the two equilibrium options for electron density really refer to how one computes $Z_{\text {eff }}$. 


\section{Quantities of Interest}

Once we have iterated the sub-densities to convergence in the Newton-Raphson solver (subject to the mixture density constraint), we can compute mixture properties using the following formulae:

$$
\begin{aligned}
P & =\sum_{i=1}^{N} y_{i} \frac{\partial v_{i}}{\partial v} P_{i}\left(\rho_{i}, T\right) \\
e & =\sum_{i=1}^{N} y_{i} e_{i}\left(\rho_{i}, T\right) \\
\frac{\partial P}{\partial T} & =\sum_{i=1}^{N} y_{i} \frac{\partial v_{i}}{\partial v} \frac{\partial P_{i}\left(\rho_{i}, T\right)}{\partial T}+\sum_{i=1}^{N} y_{i} \frac{\partial v_{i}}{\partial v} \frac{\partial v_{i}}{\partial T} \frac{\partial P_{i}\left(\rho_{i}, T\right)}{\partial v_{i}} \\
\frac{\partial e}{\partial T} & =\sum_{i=1}^{N} y_{i} \frac{\partial e_{i}\left(\rho_{i}, T\right)}{\partial T}+\sum_{i=1}^{N} y_{i} \frac{\partial v_{i}}{\partial T} \frac{\partial e_{i}\left(\rho_{i}, T\right)}{\partial v_{i}} \\
\frac{\partial P}{\partial v} & =\sum_{i=1}^{N} y_{i}\left(\frac{\partial v_{i}}{\partial v}\right)^{2} \frac{\partial P_{i}\left(\rho_{i}, T\right)}{\partial v_{i}} \\
\frac{\partial e}{\partial v} & =\sum_{i=1}^{N} y_{i} \frac{\partial v_{i}}{\partial v} \frac{\partial e_{i}\left(\rho_{i}, T\right)}{\partial v_{i}},
\end{aligned}
$$

where $P$ is the mixture pressure and $e$ is the mixture specific internal energy (energy/mass). Eqs. 3 and 4 constitute the physics, while eqs. 5,8 follow purely from the functional dependence of $P$ and $e$ on the independent variables. Also, since the thermodynamic tables use $\rho_{i}$ as an independent variable, rather than $v_{i}$, the following formulas can be substituted above:

$$
\begin{aligned}
\frac{\partial X_{i}}{\partial v_{i}} & =\frac{\partial X_{i}}{\partial\left(1 / \rho_{i}\right)}=-v_{i}^{2} \frac{\partial X_{i}}{\partial \rho_{i}} \\
\frac{\partial X}{\partial v} & =\frac{\partial X}{\partial(1 / \rho)}=-v^{2} \frac{\partial X}{\partial \rho} .
\end{aligned}
$$

Let us consider for a moment how we would derive equations for $\partial P / \partial T$ and $\partial P / \partial v$. The derivations for $\partial e / \partial T$ and $\partial e / \partial v$ will follow by analogy. Since $P_{i}$ is a function of $\rho_{i}$ (or $v_{i}$ ) and $T$, we have

$$
\Delta P_{i}=\frac{\partial P_{i}}{\partial T} \Delta T+\frac{\partial P_{i}}{\partial v_{i}} \Delta v_{i}
$$

Using the definition of pressure given by eq. 3] we see that if we multiply eq. 11 by $y_{i} \partial v_{i} / \partial v$ and sum over all tables, the result is

$$
\Delta P=\Delta T \sum_{i=1}^{N} y_{i} \frac{\partial v_{i}}{\partial v} \frac{\partial P_{i}}{\partial T}+\sum_{i=1}^{N} y_{i} \frac{\partial v_{i}}{\partial v} \frac{\partial P_{i}}{\partial v_{i}} \Delta v_{i} .
$$

Dividing this last equation by $\Delta T$ and taking the limit as the different $\Delta$ 's $\rightarrow 0$ gives eq. 5 Note that we can't simply drop the second term on the right hand side of 5. That is, even though $\partial P / \partial T$ is taken at constant $v$, the constituent specific volumes can still have changes with respect to temperature. On the other hand, $\partial P / \partial v$ is taken at constant temperature. Thus, when we divide eq. 12 by $\Delta v$, we can set $\Delta T=0$. Taking the limit as the remaining $\Delta$ 's $\rightarrow 0$ gives the desired expression for $\partial P / \partial v$.

The final result we need is to develop expressions for $\partial v_{i} / \partial v$ and $\partial v_{i} / \partial T$. We begin by assuming $f_{j}=f_{j}\left(v_{j}, T\right)$. What follows is

$$
\Delta f_{j}=\frac{\partial f_{j}}{\partial T} \Delta T+\frac{\partial f_{j}}{\partial v_{j}} \Delta v_{j}
$$


Since $\sum_{j=1}^{N} y_{j} v_{j}=v$, we also have that

$$
\Delta v=\sum_{j=1}^{N} y_{j} \Delta v_{j}
$$

Solving eq. 13 for $\Delta v_{j}$ and inserting this into eq. 14 gives:

$$
\Delta v=\sum_{j=1}^{N} \frac{y_{j} \Delta f_{j}}{\frac{\partial f_{j}}{\partial v_{j}}}-\Delta T \sum_{j=1}^{N} \frac{y_{j} \frac{\partial f_{j}}{\partial T}}{\frac{\partial f_{j}}{\partial v_{j}}} .
$$

As we are iterating until all the $f$ 's are equal, we can pull the $\Delta f_{j}$ out of the summation, call it $\Delta f$, and then re-express this using eq. 13 where we let $j \rightarrow i$. The result of these manipulations is:

$$
\Delta v=\left(\frac{\partial f_{i}}{\partial T} \Delta T+\frac{\partial f_{i}}{\partial v_{i}} \Delta v_{i}\right) \sum_{j=1}^{N} \frac{y_{j}}{\frac{\partial f_{j}}{\partial v_{j}}}-\Delta T \sum_{j=1}^{N} \frac{y_{j} \frac{\partial f_{j}}{\partial T}}{\frac{\partial f_{j}}{\partial v_{j}}} .
$$

Now divide eq. 16 by $\Delta v$ (assuming temperature is constant) and solve for $\partial v_{i} / \partial v$. Then divide eq. 16 by $\Delta T$ and solve for $\partial v_{i} / \partial T$. The desired quantities are:

$$
\begin{aligned}
& \left.\frac{\partial v_{i}}{\partial v}\right)_{T} \equiv \frac{\partial v_{i}}{\partial v}=\frac{\left(\frac{\partial f_{i}}{\partial v_{i}}\right)^{-1}}{\sum_{j=1}^{N} y_{j}\left(\frac{\partial f_{j}}{\partial v_{j}}\right)^{-1}} \\
& \left.\frac{\partial v_{i}}{\partial T}\right)_{v} \equiv \frac{\partial v_{i}}{\partial T}=\frac{\partial v_{i}}{\partial v} \sum_{j=1}^{N} y_{j} \frac{\partial f_{j}}{\partial T}\left(\frac{\partial f_{j}}{\partial v_{j}}\right)^{-1}-\frac{\partial f_{i}}{\partial T}\left(\frac{\partial f_{i}}{\partial v_{i}}\right)^{-1} .
\end{aligned}
$$

As a sanity check, we observe from the previous two equations that

$$
\left.\sum_{i=1}^{N} y_{i}\left(\frac{\partial v_{i}}{\partial v}\right)_{T}=\frac{\partial v}{\partial v}\right)_{T}=1
$$

as well as

$$
\left.\sum_{i=1}^{N} y_{i}\left(\frac{\partial v_{i}}{\partial T}\right)_{v}=\frac{\partial v}{\partial T}\right)_{v}=0
$$

The reason we can take $y_{i}$ inside the differential is that the isotopics are fixed during the Newton-Raphson procedure. Hence, the masses of the different isotopes (and therefore the $y_{i}$ 's) are constant. 


\section{Application}

The above discussion has shown at a high level how to bring an atomic mixture with a known mixture density, temperature, and isotopics into thermodynamic equilibrium and how to compute mixture thermodynamic quantities of interest. For example, using eqs. 5, 8 we can construct derived quantities like sound speed, bulk modulus, etc. . We will not delve into the minutiae of how the Newton-Raphson procedure conducts its iterations, however, the choice of quantities to iterate on warrants some discussion. The EOS tables are constructed using density and temperature as independent variables and so the straightforward approach is to do forward lookups of $f$ using the current values for the sub-densities ( $\rho_{i}$ 's) and the known temperature. Then we develop a way of adjusting the changes in the sub-densities to arrive at better guesses for the next iteration. An alternative procedure is to iterate on the single quantity $f$ by performing reverse or inverse lookups. By reverse lookups, we mean that we interrogate the table for $\rho_{i}(f, T)$ instead of the forward lookup procedure which gives $f\left(\rho_{i}, T\right)$. The advantage with the reverse lookups is that we are only iterating on a single quantity, $f$, which is adjusted until the mixture density constraint is satisfied. The disadvantage is that iteration is necessary to provide the reverse lookup from the table. Also, forward and reverse lookups are not necessarily compatible or consistent. Therefore, we have decided in the current implementation to iterate on more quantities by using forward lookups, even though when more than two tables are involved, it may be more difficult to reach a converged equilibrium state.

It is often desired to compute the temperature from the mixture internal energy and density. For simplicity, let's assume that we have modified the internal energy, but the density has remained fixed. The procedure we use for the atomic mixture is to adjust the temperature and do forward lookups of energy until we satisfy the constraint that:

$$
e(\rho, T)=\sum_{i=1}^{N} y_{i} e_{i}\left(\rho_{i}, T\right) .
$$

In the Lagrange hydro, we have the more complicated circumstance where both the material density and internal energy are being updated in the $3^{\text {rd }}$ order Runge-Kutta time integration scheme. The sub-densities that are used in the above constraint, however, will sum to the old mixture density and not the new density. Also, we can't simply re-equilibrate the mixture with the modified density to find the new sub-densities, since the needed temperature is unknown. Fortunately, there are a few ways out of this chicken and egg dilemma. The most computationally expensive way (and probably the most accurate as well) is to do a double iteration on the sub-densities and temperature, such that after we are equilibrated, we also satisfy the density and energy constraints. A simpler procedure would be to only iterate on temperature, but to use a modified sub-density for the table lookups that is consistent with the current mixture density. Thus, we will use the following energy constraint:

$$
e(\rho, T)=\sum_{i=1}^{N} y_{i} e_{i}\left(1 / v_{i}^{*}, T\right) .
$$

There are two obvious choices for the $v_{i}^{*}$, with the first one given by the simple scaling

$$
v_{i}^{*}=v_{i}\left(\frac{v_{n e w}}{v}\right)=v_{i}+\frac{v_{i}}{v}\left(v_{n e w}-v\right) .
$$

The second approach is to make a Taylor-series in specific volume. The result is

$$
v_{i}^{*}=v_{i}+\frac{\partial v_{i}}{\partial v}\left(v_{\text {new }}-v\right) \text {. }
$$


Note that from eq. 17 and eqs. 9-10 we have the result that

$$
\frac{\partial v_{i}}{\partial v}=\frac{\left(\frac{\partial f_{i}}{\partial v_{i}}\right)^{-1}}{\sum_{j=1}^{N} y_{j}\left(\frac{\partial f_{j}}{\partial v_{j}}\right)^{-1}}=\frac{v_{i}^{2}\left(\frac{\partial f_{i}}{\partial \rho_{i}}\right)^{-1}}{\sum_{j=1}^{N} y_{j} v_{j}^{2}\left(\frac{\partial f_{j}}{\partial \rho_{j}}\right)^{-1}} .
$$

Also, since $\partial f_{i} / \partial \rho_{i} \geq 0$, we know that $0 \leq \partial v_{i} / \partial v \leq 1$. This means that all the $v_{i}^{*}$ 's will increase or decrease, depending on the sign of $v_{n e w}-v$. The advantage of the first approach is that the sub-densities will never go negative. The disadvantage to this approach is that it is essentially approximating the derivative $\partial v_{i} / \partial v$ by $v_{i} / v$, which is only zeroeth order accuarate. The Taylor series approximation is first order accurate, but has the potential of driving the sub-densities negative (particularly in the case of $v_{n e w}>>v$ ).

\section{$4 \quad$ Analytic $Z_{\text {eff }}$}

It was noted earlier that there are 2 ways of computing $Z_{\text {eff }}$ when we want to use the electron density equilibrate option. One way is to simply do a forward table lookup of $Z_{\text {eff }}$ based on $\rho_{i}$ and $T$. The table also provides values for $\partial Z_{e f f} / \partial \rho$ and $\partial Z_{e f f} / \partial T$. The alternative is to use an analytic data fit for $Z_{e f f}$ developed by Dick More based on Thomas-Fermi theory. The inputs for the analytic formula are $T(K e V)$, $\rho_{i}\left(\mathrm{~g} / \mathrm{cm}^{3}\right),\langle z\rangle_{i}$, and $\langle\hat{z}\rangle_{i}$. Here, $\langle z\rangle_{i}$, and $\langle\hat{z}\rangle_{i}$ are given by

$$
\begin{aligned}
<z>_{i} & =\sum_{j} \chi_{j} Z_{j} \\
<\hat{z}>_{i} & =\exp \left[\frac{2}{3} \frac{z z \ln z>_{i}}{<z>_{i}}\right] \\
<z \ln z>_{i} & =\sum_{j} \chi_{j} Z_{j} \ln Z_{j},
\end{aligned}
$$

where it is understood in the above formulas that $\chi_{j}$ are number fractions and the $j$ in the summation refers to all isotopes that are associated with the $i^{\text {th }}$ table.

We start the formula for $Z_{e f f}$ by defining a scaled temperature $\bar{T}$ and a dimensionless temperature $\hat{T}$ as:

$$
\begin{aligned}
& \bar{T}=\alpha T \\
& \hat{T}=\frac{\bar{T}}{1+\bar{T}},
\end{aligned}
$$

where $\alpha=1000 /<\hat{z}>^{2}$. We then define $a_{Y}$, a function of scaled temperature and $b_{Y}$ and $c_{Y}$, two functions of dimensionless temperature by the following:

$$
\begin{aligned}
a_{Y} & =a_{1} \bar{T}^{a_{2}}+a_{3} \bar{T}^{a_{4}} \\
b_{Y} & =-\exp \left[b_{0}+b_{1} \hat{T}+b_{2} \hat{T}^{7}\right] \\
c_{Y} & =c_{1} \hat{T}+c_{2},
\end{aligned}
$$

where

$$
\begin{aligned}
& a_{1}=.003323467 \\
& a_{2}=.97183224 \\
& a_{3}=9.26148 e-05 \\
& a_{4}=3.1016524
\end{aligned}
$$




$$
\begin{aligned}
& b_{0}=-1.762999 \\
& b_{1}=1.4317567 \\
& b_{2}=0.31546338 \\
& c_{1}=-.36666667 \\
& c_{2}=.98333333 .
\end{aligned}
$$

We then define two functions $p_{1}$ and $p_{2}$ by:

$$
\begin{aligned}
p_{1} & =a_{Y}^{c_{Y}} Z_{R}^{b_{Y} c_{Y}} \rho^{b_{Y} c_{Y}} \\
p_{2} & =Z_{R}^{c_{Y}} \rho^{b_{Y} c_{Y}} \\
Z_{R} & =\frac{<z>}{A<\hat{z}>^{2 / 3}} .
\end{aligned}
$$

Finally, the relation for $Z_{e f f}$ takes the form:

$$
\begin{aligned}
Z_{e f f} & =<z>\hat{Z}_{\text {eff }} \\
\hat{Z}_{e f f} & =\frac{x}{1+x+\sqrt{1+2 x}} \\
x & =2 \gamma_{1}^{2}\left(p_{1}+p_{2}\right)^{2 \gamma_{2} / c_{Y}} \\
\gamma_{1} & =2.6752506 \\
\gamma_{2} & =0.33120023 .
\end{aligned}
$$

Now that we have a closed expression for $Z_{e f f}$, we need to take analytic derivatives of $Z_{\text {eff }}$ with respect to density and temperature. To this end, we express the density derivative using the chain rule as:

$$
\frac{\partial Z_{e f f}}{\partial \rho}=\frac{\partial Z_{e f f}}{\partial \hat{Z}_{e f f}} \frac{\partial \hat{Z}_{e f f}}{\partial x} \frac{\partial x}{\partial \rho} .
$$

Using simple calculus, we obtain:

$$
\begin{aligned}
\frac{\partial \hat{Z}_{e f f}}{\partial x} & =x \frac{d}{d x}(1+x+\sqrt{1+2 x})^{-1}+(1+x+\sqrt{1+2 x})^{-1} \\
& =\frac{\hat{Z}_{e f f}}{x}\left[1-\hat{Z}_{e f f}\left(1+\frac{1}{\sqrt{1+2 x}}\right)\right], \\
\frac{\partial x}{\partial \rho} & =\frac{4 \gamma_{1}^{2} \gamma_{2}}{c_{Y}}\left(p_{1}+p_{2}\right)^{2 \gamma_{2} / c_{Y}-1}\left(\frac{\partial p_{1}}{\partial \rho}+\frac{\partial p_{2}}{\partial \rho}\right) \\
& =\frac{2 \gamma_{2} x\left(b_{Y} p_{1}+p_{2}\right)}{\left(p_{1}+p_{2}\right) \rho} \\
\frac{\partial Z_{e f f}}{\partial \hat{Z}_{e f f}} & =<z>.
\end{aligned}
$$

Therefore we obtain the relatively straightforward result that

$$
\frac{\partial Z_{e f f}}{\partial \rho}=\frac{2 \gamma_{2} Z_{e f f}\left(b_{Y} p_{1}+p_{2}\right)}{\left(p_{1}+p_{2}\right) \rho}\left[1-\hat{Z}_{e f f}\left(1+\frac{1}{\sqrt{1+2 x}}\right)\right] .
$$

Proceeding in a similar fashion, we can derive the following result for the derivative of $Z_{\text {eff }}$ with respect to temperature.

$$
\frac{\partial Z_{e f f}}{\partial T}=\frac{\partial Z_{e f f}}{\partial \hat{Z}_{e f f}} \frac{\partial \hat{Z}_{e f f}}{\partial x} \frac{\partial x}{\partial T}
$$


If we take the natural $\log$ of eq. 28 (the definition for $x$ ) and then differentiate both sides with respect to temperature, we obtain:

$$
\frac{\partial x}{\partial T}=\frac{2 \gamma_{2} x}{c_{Y}}\left[\frac{\partial p_{1} / \partial T+\partial p_{2} / \partial T}{p_{1}+p_{2}}-\frac{\ln \left(p_{1}+p_{2}\right)}{c_{Y}} \frac{\partial c_{Y}}{\partial T}\right]
$$

Equation 31 now assumes the form of:

$$
\frac{\partial Z_{e f f}}{\partial T}=\frac{2 \gamma_{2} Z_{e f f}}{c_{Y}}\left[1-\hat{Z}_{e f f}\left(1+\frac{1}{\sqrt{1+2 x}}\right)\right]\left[\frac{\partial p_{1} / \partial T+\partial p_{2} / \partial T}{p_{1}+p_{2}}-\frac{\ln \left(p_{1}+p_{2}\right)}{c_{Y}} \frac{\partial c_{Y}}{\partial T}\right] .
$$

To find $\partial p_{1} / \partial T$ and $\partial p_{2} / \partial T$, we use the same trick as before. That is, we take the natural log of eqs. 26, 27 and differentiate both sides with respect to temperature. The result is:

$$
\begin{aligned}
\frac{\partial p_{1}}{\partial T} & =p_{1}\left\{c_{Y}\left[\frac{1}{a_{Y}} \frac{\partial a_{Y}}{\partial T}+\frac{\partial b_{Y}}{\partial T} \ln \bar{Z}_{R}\right]+\frac{\partial c_{Y}}{\partial T}\left[\ln a_{Y}+b_{Y} \ln \bar{Z}_{R}\right]\right\} \\
\frac{\partial p_{2}}{\partial T} & =p_{2} \frac{\partial c_{Y}}{\partial T} \ln \bar{Z}_{R} \\
\bar{Z}_{R} & \equiv \rho Z_{R} .
\end{aligned}
$$

Now we simply need derivatives of $a_{Y}, b_{Y}$, and $c_{Y}$ with respect to temperature to finish the derivation.

$$
\begin{aligned}
\frac{\partial a_{Y}}{\partial T} & =\frac{\alpha}{\bar{T}}\left(a_{1} a_{2} \bar{T}^{a_{2}}+a_{3} a_{4} \bar{T}^{a_{4}}\right) \\
\frac{\partial b_{Y}}{\partial T} & =\frac{\partial b_{Y}}{\partial \hat{T}} \frac{\partial \hat{T}}{\partial \bar{T}} \frac{\partial \bar{T}}{\partial T} \\
\frac{\partial c_{Y}}{\partial T} & =\frac{\partial c_{Y}}{\partial \hat{T}} \frac{\partial \hat{T}}{\partial \bar{T}} \frac{\partial \bar{T}}{\partial T} \\
\frac{\partial b_{Y}}{\partial \hat{T}} & =b_{Y}\left(b_{1}+7 b_{2} \hat{T}^{6}\right) \\
\frac{\partial c_{Y}}{\partial \hat{T}} & =c_{1} \\
\frac{\partial \hat{T}}{\partial \bar{T}} \frac{\partial \bar{T}}{\partial T} & =\alpha \frac{\hat{T}(1-\hat{T})}{\bar{T}} .
\end{aligned}
$$

Finally, recall that it is really the electron density that is put into equilibrium and not the effective charge. Therefore, from the perspective of the Newton-Raphson procedure, what we really need are the derivatives of electron density with respect to density and temperature. Due to the simple relation between $n_{e}$ and $Z_{e f f}$, the desired derivatives are:

$$
\begin{aligned}
\frac{\partial n_{e}}{\partial \rho} & =\frac{\rho}{A} \frac{\partial Z_{e f f}}{\partial \rho}+\frac{Z_{e f f}}{A} \\
\frac{\partial n_{e}}{\partial T} & =\frac{\rho}{A} \frac{\partial Z_{e f f}}{\partial T} .
\end{aligned}
$$

\section{Thermodynamic Consistency}

It was mentioned previously that the relations for expressing the mixture pressure and energy in terms of its constituents come from physics, while their derivatives are purely the result of mathematics. An interesting consequence of a more fundamental approach is that we can check the validity of the relations 
for the mixture energy and pressure by working with $a$, the specific Helmholtz free energy and $s$, the specific entropy.

For example, the mixture free energy can be expressed as:

$$
a=e-T s .
$$

Now we assume a specific form for the constituent free energies, $a_{i}$, and how they are related to the mixture free energy.

$$
\begin{aligned}
a_{i} & =e_{i}-T s_{i} \\
a & =\sum_{i=1}^{N} y_{i} a_{i}=\sum_{i=1}^{N} y_{i}\left(e_{i}-T s_{i}\right) .
\end{aligned}
$$

The first law of thermodynamics can be expressed as

$$
d e=T d s-P d v
$$

Taking the differential of eq. 34 and making use of the first law relation gives:

$$
d a=-s d T-P d v
$$

which implies that

$$
\begin{aligned}
-s & \equiv\left(\frac{\partial a}{\partial T}\right)_{v} \\
-P & \equiv\left(\frac{\partial a}{\partial v}\right)_{T} .
\end{aligned}
$$

Differentiating eq. 36 with respect to temperature gives:

$$
-s=\sum_{i=1}^{N} y_{i}\left[\left(\frac{\partial e_{i}}{\partial T}-T \frac{\partial s_{i}}{\partial T}\right)-s_{i}+\left(\frac{\partial e_{i}}{\partial v_{i}}-T \frac{\partial s_{i}}{\partial v_{i}}\right) \frac{\partial v_{i}}{\partial T}\right] .
$$

To simplify this equation for the entropy, let's express the first law for a particular component in the mixture:

$$
d e_{i}=T d s_{i}-P_{i} d v_{i}
$$

Dividing eq. 40 by $d T$ (at constant $v_{i}$ ) gives the result

$$
\left(\frac{\partial e_{i}}{\partial T}-T \frac{\partial s_{i}}{\partial T}\right)=0
$$

Similarly, if we divide eq. 40 by $d v_{i}$ (at constant temperature) we arrive at

$$
\left(\frac{\partial e_{i}}{\partial v_{i}}-T \frac{\partial s_{i}}{\partial v_{i}}\right)=-P_{i}
$$

Inserting these last two results into eq. 39 gives:

$$
s=\sum_{i=1}^{N} y_{i}\left[s_{i}+P_{i} \frac{\partial v_{i}}{\partial T}\right]
$$


Now that we have relations for $a$ and $s$ in terms of their constituents, we can use eq. 34 to solve for $e$. The result is:

$$
e=\sum_{i=1}^{N} y_{i}\left[e_{i}+T P_{i} \frac{\partial v_{i}}{\partial T}\right] .
$$

Thus, the specific internal energy is not simply a sum of its parts. As John Castor points out in his memo on Mixing Free-Energy Based EOS Models, this is probably not a good thing. However, for the case of total pressure equilibration, all of the $P_{i}$ are equal. This means that the pressure can be pulled out of the above summation and that this term is identically zero, from eq. 20. So for the case of total pressure mixing, we do recover the result that the mixture energy is a simple sum of its parts. Note that by using the fundamental definition for pressure we can show that:

$$
P \equiv-\left(\frac{\partial a}{\partial v}\right)_{T}=\sum_{i=1}^{N} y_{i} \frac{\partial v_{i}}{\partial v} P_{i}
$$

which is identical to eq. 3. Thus, a rigorous derivation of the mixture pressure and energy shows that we have a discrepency in the energy definition for the case of equilibrating a quantity other than totoal pressure. We will focus our future work on assessing what consequences there are to omitting this extra term in the mixture energy definition for the cases of electron density and chemical potential equilibration.

\section{Test Case}

For the case of an atomic mixture of two ideal gases that are equilibrated under the total pressure option, there exists an analytic solution for the sub-densities and the mixture pressure. Thus we can run a simple test case at a variety of densities and temperatures to verify that the Newton-Raphson method converges to the analytic solution. Let the ideal gas mixture have the following number fractions for its isotopics: $n_{D}=.25$, $n_{T}=.25, n_{N 14}=.25, n_{O 16}=.25$. We will also let the subscript 1 refer to the light gas (the part of the mixture made up of the hydrogen isotopes) and the subscript 2 refer to the heavier isotopes. Converting the number fractions into mass fractions gives $y_{1}=.144$ and $y_{2}=.856$. Note that for this example, we are using analytic equations of state to represent the two gases, rather than tabular ones. At equilibrium, we will have:

$$
P=P_{1}=P_{2}=\frac{\rho_{1} R_{u} T}{A_{1}}=\frac{\rho_{2} R_{u} T}{A_{2}}
$$

where $R_{u}$ is the universal gas constant and $A_{i}$ are the average molecular weights of the two sub-gases. Using the mixture density constraint gives 2 equations in 2 unknowns for $\rho_{1}$ and $\rho_{2}$. The solution is:

$$
\begin{aligned}
\rho_{1} & =\rho\left(y_{1}+y_{2} \frac{A_{1}}{A_{2}}\right) \\
\rho_{2} & =\rho\left(y_{2}+y_{1} \frac{A_{2}}{A_{1}}\right) .
\end{aligned}
$$

Figure 1 shows the mixture pressure (computed analytically and computed after the Newton-Raphson procedure using the summation formula for the mixture pressure given earlier in the text) as a function of density for a fixed temperature of $1 \mathrm{KeV}$. Figure 2 shows pressure vs. temperature at a fixed mixture density of $.001 \mathrm{~g} / \mathrm{cm}^{3}$. In both figures, we see that there is excellent agreement (to approximately 9 decimal places) between the analytically computed pressure and the pressure computed using eq. 3 


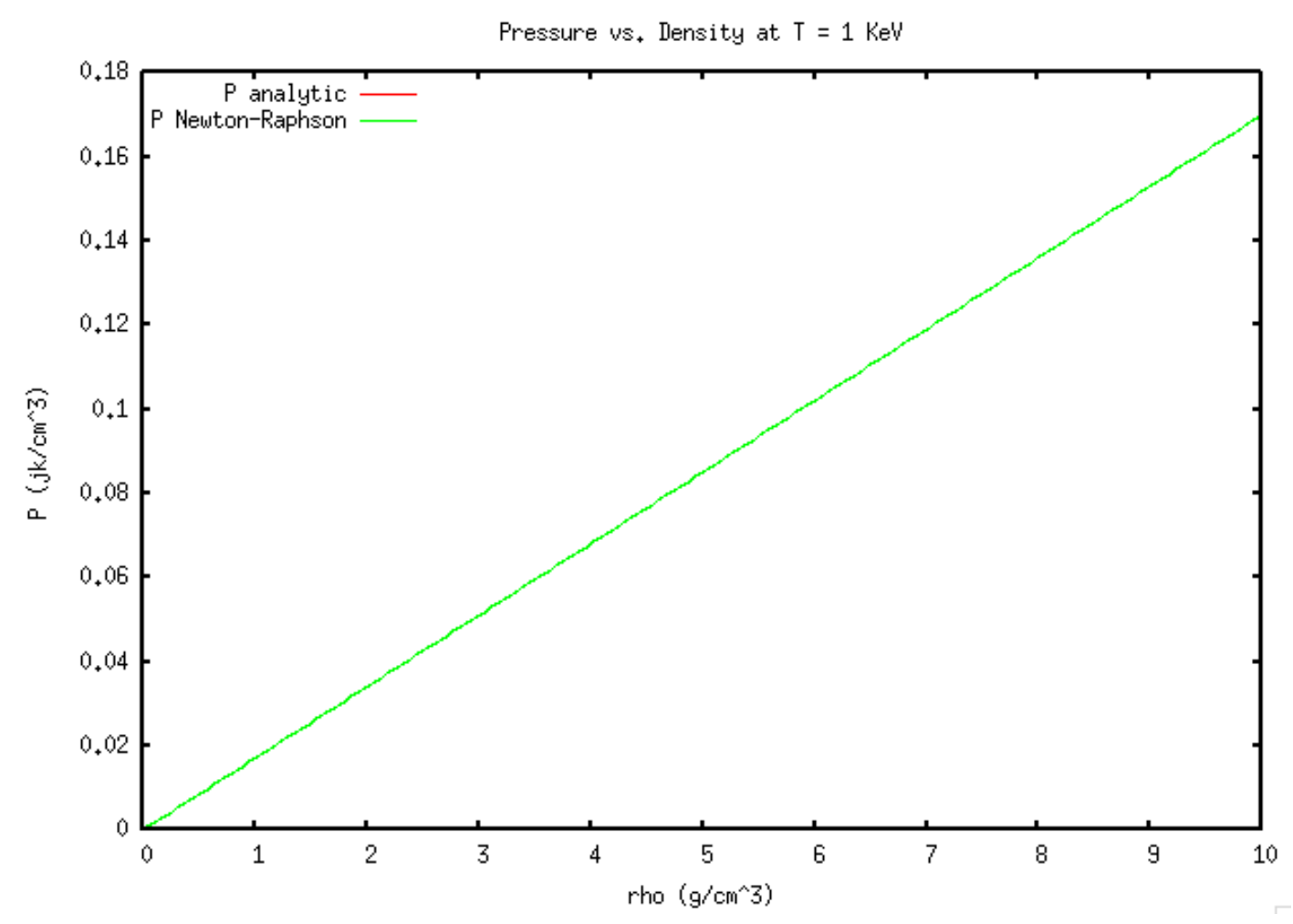

Figure 1: Pressure vs Density at $T=1 \mathrm{KeV}$. 


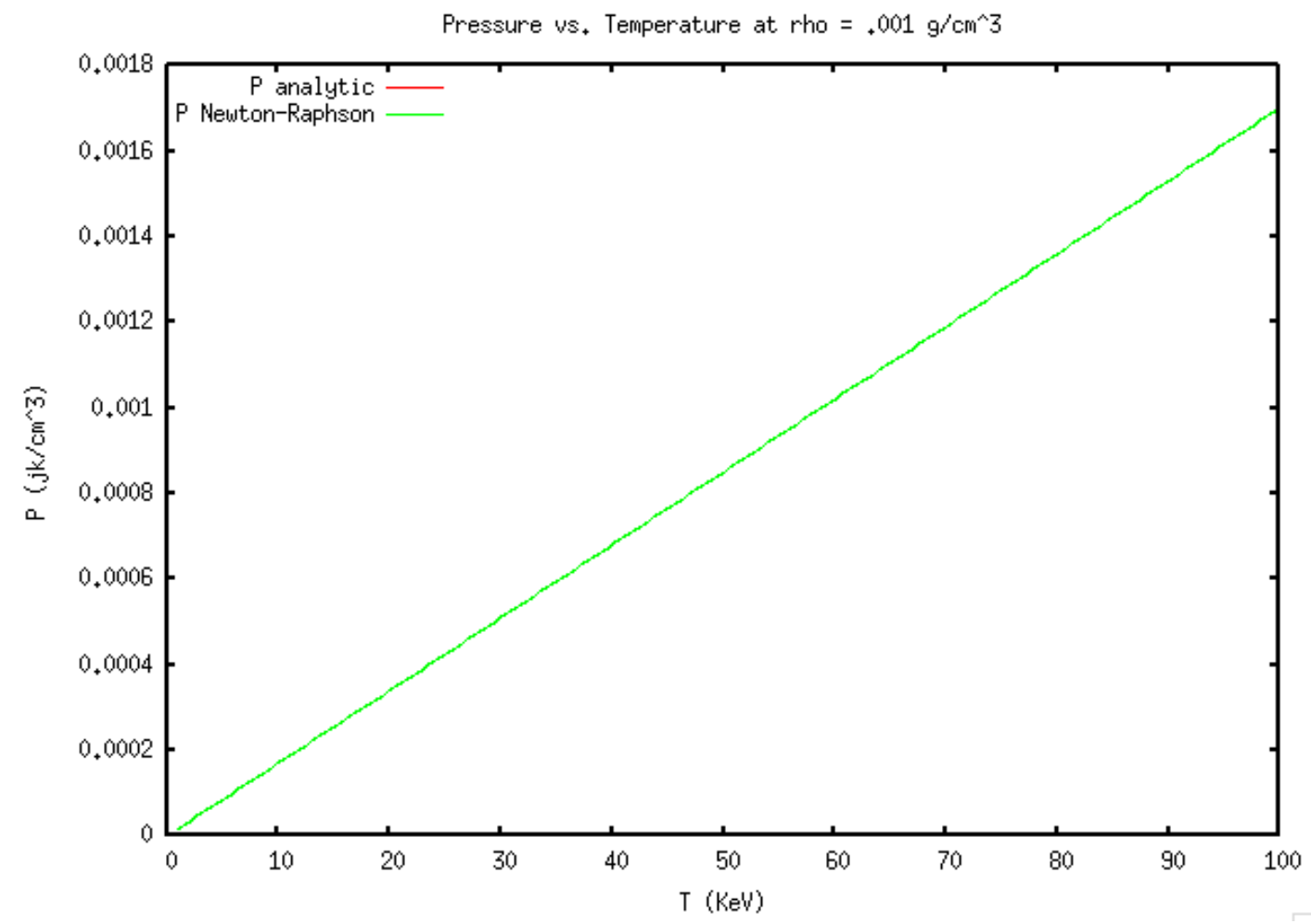

Figure 2: Pressure vs Temperature at $\rho=.001 \mathrm{~g} / \mathrm{cm}^{3}$. 